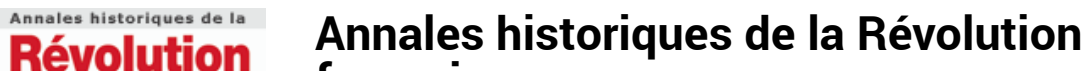

française française

333 | juillet-septembre 2003

Varia

\section{Guillaume MÉTAIRIE, Des juges de proximité: les juges de paix. Biographies parisiennes (1790-1838)}

\section{Claude Coquard}

\section{(2) OpenEdition \\ 1 Journals}

\section{Édition électronique}

URL : https://journals.openedition.org/ahrf/10809

DOI : 10.4000/ahrf.10809

ISSN : 1952-403X

Éditeur :

Armand Colin, Société des études robespierristes

\section{Édition imprimée}

Date de publication : 1 septembre 2003

ISSN : 0003-4436

\section{Référence électronique}

Claude Coquard, « Guillaume MÉTAIRIE, Des juges de proximité : les juges de paix. Biographies

parisiennes (1790-1838) », Annales historiques de la Révolution française [En ligne], 333 | juillet-septembre 2003, mis en ligne le 07 décembre 2009, consulté le 23 avril 2022. URL : http://

journals.openedition.org/ahrf/10809 ; DOI : https://doi.org/10.4000/ahrf.10809

Ce document a été généré automatiquement le 23 avril 2022.

Tous droits réservés 


\title{
Guillaume MÉTAIRIE, Des juges de proximité: les juges de paix. Biographies parisiennes (1790-1838)
}

\author{
Claude Coquard
}

\section{RÉFÉRENCE}

Guillaume MÉTAIRIE, Des juges de proximité : les juges de paix. Biographies parisiennes

(1790-1838), Paris, L'Harmattan, 2002, 261 p., ISBN : 2-7475-3949-6, $23 €$.

1 Huit ans après la parution de l'ouvrage qu'il a consacré au Monde des juges de paix de Paris, 1790-1838, nourri de la thèse d'État qu'il soutint précédemment sur cet objet, Guillaume Métairie nous offre un ensemble prosopographique concernant 211 des acteurs essentiels de cette justice de proximité qui fonctionna dans la capitale entre les premières élections de l'automne 1790 et la réforme mise en place en 1838 sous la monarchie de Juillet. Emile Ducoudray a présenté dans notre revue une analyse complète du premier ouvrage (A.H.R.F., $n^{\circ} 306$, octobre-décembre 1996) et c'est dans sa continuité que s'inscrit le présent compte rendu.

Dans son introduction, l'auteur, après avoir rappelé les principales étapes de la mise en place et des modifications, juridiques et politiques, que l'institution connut au cours des cinquante premières années de son existence, précise à quelles sources il a puisé sa documentation et pour quel objectif il a décidé de compléter ici son travail antérieur. On retrouve la totalité des outils utilisés dans la thèse auxquels ont été ajoutés un certain nombre de précisions tirées notamment de l'exploitation de minutes notariales, le tout constituant une riche information dont témoignent les près de 2000 notes infrapaginales de l'ouvrage.

3 En raison de l'extrême diversité des renseignements que l'auteur a pu extraire des sources exploitées - et des évidentes lacunes que celles-ci comportent -, le lecteur ne trouvera ici aucune homogénéité dans la présentation des portraits de ces 211 juges de 
paix parisiens. Tel d'entre eux, comme Jacques-René Mortier, qui pourtant occupa le siège de la section du Jardin-des-Plantes de l'origine jusqu'aux bouleversements de l'été 1792, n'occupe que cinq lignes, quand son collègue Jean Manjaud de la section des Tuileries, titulaire du poste pendant la même période, a droit à trois pages entières : celui-ci offre l'image d'un engagement politique particulièrement courageux au cours du printemps et de l'été 1792 qui lui valut les foudres du Tribunal révolutionnaire et une véritable proscription pendant la Terreur alors que celui-là ne semble pas avoir pris les mêmes risques ni fait l'objet des mêmes poursuites. La même hétérogénéité préside à la mise en place de l'état civil des différents personnages: Jean-Philippe Normand, juge de paix du $\mathrm{XI}^{\mathrm{e}}$ arrondissement peut être suivi de sa naissance à son décès, dans son itinéraire socioprofessionnel comme dans ses diverses domiciliations du 29 janvier 1766 jusqu'au 21 avril 1820, alors que la vie de son collègue du $\mathrm{X}^{\mathrm{e}}$ arrondissement, Nicolas Thomassin de la Fortelle, reste à peu près ignorée. Enfin, et pour les mêmes raisons, les éléments patrimoniaux - qui pourraient donner lieu à la mise en lumière d'une certaine typologie sociale de ces acteurs principaux de la justice de paix parisienne - ne sont que fragmentairement et occasionnellement rappelés.

Confirmant les conclusions présentées dans sa thèse, Guillaume Métairie présente les détails d'une galerie de portraits, organisée alphabétiquement, et qui fait bien apparaître l'ambiguïté de la situation professionnelle de ces juges de paix parisiens constamment soumis aux aléas des politiques successives du pouvoir.

5 À l'exception de cinq d'entre eux, tous se trouvent évincés de leur responsabilité judiciaire à la suite des événements parisiens du 10 août 1792 et de l'épuration à laquelle procède la Commune de Paris à leur égard. De même, pendant tout le Directoire, les élections connaissent de nombreuses remises en cause et la nomination par le pouvoir se substitue souvent à l'expression démocratique du vote. Certains de ces magistrats connaissent - parfois à plusieurs reprises à l'instar de Mathurin Bouin, juge de paix de la section du Marché en août 1792 - les rigueurs de l'arrestation voire de la condamnation 4 mort. La réduction de 48 à 12 sièges des justices de paix parisiennes entraîne l'élimination de nombreux anciens juges de paix qui, au mieux, peuvent retrouver un siège de juge suppléant selon le bon vouloir du Premier Consul. Et les choix de l'Empire, comme ceux de la Restauration ou de la monarchie de Juillet connaissent les mêmes marques du politiquement correct de l'époque.

6 Ces perturbations politiques se retrouvent dans le nombre élevé des titulaires successifs des sièges entre la mise en place de l'institution et la suppression de fait de l'élection de ces magistrats en l'an X. Seule la section de l'Hôtel de Ville connaît une exceptionnelle continuité en la personne de Léon-Toussaint Wisnick: il dirigea les audiences - à l'exception de la période de sa brève incarcération entre le 12 mai et le 26 août 1794 - de janvier 1791 jusqu'à sa mort en septembre 1815, et fut constamment réélu puis désigné à son poste. Toute autre est la situation de la section de La-FontaineGrenelle où se succédèrent, entre 1791 et 1799, dix juges de paix (trois d'entre eux retrouvant à échéance leur siège). Le recensement exhaustif fait donc apparaître une fréquence moyenne égale à 4,2 juges par siège entre 1791 et les dernières élections directes de l'an X, en février 1802 (3,7 si l'on tient compte du fait que certains des titulaires furent réélus à une ou deux reprises pendant cette période). Au cours des 36 années suivantes de fonctionnement de l'institution jusqu'à la grande réforme de 1838, les 12 arrondissements de Paris connaissent une fréquence moyenne de remplacement 
des juges de paix égale à 4 , témoignant par là même d'une stabilisation plus grande qu'au cours de la période précédente.

7 La mise en scène organisée par Guillaume Métairie de ces juges de paix parisiens permet de saisir l'extrême diversité de ces personnages. C'est Augustin Duchesne de la section Popincourt présenté dans un rapport du comité civil en novembre 1794, en pleine réaction anti-robespierriste, comme « fort sujet à s'enivrer (...) loin de ramener le calme et de remettre les choses à leur juste valeur, il n'a jamais montré que des principes incendiaires », et dont certains de ses assesseurs l'auraient décrit comme « ivrogne, emporté, méchant, factieux ». C'est l'intermittent Antoine Thorillon dont l'enthousiasme révolutionnaire fraîchit au gré des aléas politiques et qui s'accroche désespérément à son siège de juge de paix en dépit des doutes que l'administration peut émettre sur son compte et de son état de santé chancelant. C'est l'intrigant François-Stanislas Chauvet dont l'entregent auprès de la Chancellerie lui permet d'accéder, après de longs efforts diplomatiques, au siège de juge de paix du VII ${ }^{e}$ arrondissement rendu libre par le retrait volontaire... de son beau père, Jean Odent. Et c'est encore, entre autres, la lumineuse figure de Léon-Toussaint Wisnick, déjà cité, et dont la longévité comme juge de paix pendant toute la Révolution et la période impériale s'explique par des qualités personnelles et intellectuelles reconnues par le préfet Frochot qui l'estimait « instruit, plein de zèle, affable ».

Synthétisant ces différents aperçus biographiques, le Registre territorial dressé avec minutie par l'auteur pour les 48 sièges de la période révolutionnaire et jusqu'à l'an $\mathrm{X}$, comme pour les justices de paix des 12 arrondissements parisiens qui organisent l'espace judiciaire à partir de 1802, est un précieux outil de travail pour toute recherche ultérieure sur le fonctionnement de l'institution pendant cette période. Le chercheur pourrait être intéressé par la mise en place d'un tableau récapitulatif des origines géographiques, socioprofessionnelles et politiques de ces "magistrats de proximité ». Quelques comparaisons possibles avec des études déjà avancées sur le personnel des justices de paix dans d'autres espaces - par exemple, le D.E.A. de «Droit et science politique " de Nicolas Delaselle, en 1993, Le personnel des justices de paix dans l'Yonne pendant la Révolution (1790-An XI), les travaux d'Anthony Crubaugh résumés, en 2000, dans son livre Balancing the Scales of Justice. Local Courts and Rural Society in Southwest France (1750-1800) - auraient permis de témoigner encore plus précisément de la spécificité de ces juges de paix parisiens particulièrement soumis aux vicissitudes de la vie politique à la fin du XVIII ${ }^{\mathrm{e}}$ siècle et dans le premier tiers du XIX ${ }^{\mathrm{e}}$ siècle. L'abondante bibliographie aurait peut-être pu donner place à ces recherches actuelles - et à d'autres encore - comme elle aurait pu comporter au moins une référence au numéro 11 Paris de l'Atlas de la Révolution française dont une ou deux cartes auraient pu être reproduites, avec l'accord des auteurs, Emile Ducoudray, Raymonde Monnier et Daniel Roche, afin d'éclairer plus nettement la localisation de l'institution dans l'espace parisien du temps. La conclusion de l'ouvrage de Guillaume Métairie par un index précis ajoute, s'il en était besoin, aux qualités pédagogiques et intellectuelles d'un outil de référence incontournable et dont l'intérêt, comme l'indique justement l'auteur, ne manque pas d'opportunité dans les débats actuels sur l'importance des « justices de proximité ». 